\title{
Audit of prehospital thrombolysis by general practitioners in peripheral practices in Grampian
}

\author{
J Rawles, C Sinclair, K Jennings, L Ritchie, N Waugh
}

\begin{abstract}
Background-In the Grampian region early anistreplase trial (GREAT), domiciliary thrombolysis by general practitioners was associated with a halving of one year mortality compared with hospital administration. However, after completion of the trial and publication of the results, the use of this treatment by general practitioners declined sharply. Objective-To increase the proportion of eligible patients receiving timely thrombolytic treatment from their general practitioners.
\end{abstract}

Setting-Practices in Grampian located $\geqslant 30$ minutes' travelling time from Aberdeen Royal Infirmary, where patients with suspected acute myocardial infarction were referred after being seen by general practitioners.

Audit standard-A call-to-needle time of 90 minutes, as proposed by the British Heart Foundation (BHF).

Methods-Findings of this audit of prehospital management of acute myocardial infarction were periodically fed back to the participating doctors, when practice case reviews were also conducted.

Results-Of 414 administrations of thrombolytic treatment, $146(35 \%)$ were given by general practitioners and 268 (65\%) were deferred until after hospital admission. Median call-to-needle times were $45(94 \% \leqslant 90)$ and $145(7 \% \leqslant 90)$ minutes, respectively. Survival at one year was improved with prehospital compared with hospital thrombolysis $(83 \%$ v $73 \%$; $p<0.05)$. The proportion of patients receiving thrombolytic treatment from their general practitioners did not increase during the audit.

Conclusions-In practices $\geqslant 30$ minutes from hospital, the BHF audit standard was readily achieved if general practitioners gave thrombolytic treatment, but not otherwise. Knowledge of the benefits of early thrombolysis, and feedback of audit results, did not lead to increased prehospital thrombolytic use. Additional incentives are required if general practitioners are to give thrombolytic treatment. (Heart 1998;80:231-234)

Keywords: thrombolysis; general practitioners; prehospital care; acute myocardial infarction

Dr J Rawles, Medicin

Assessment Research Unit,

Aberdeen Royal Infirmary,

Foresterhill, Aberdeen AB25

2ZN, UK

Accepted for publication 27 May 1998 In the Grampian region early anistreplase tria (GREAT) it was shown that it is feasible and safe for general practitioners to give thrombo- lytic treatment. ${ }^{1}$ The 29 practices that participated were located $\geqslant 30$ minutes' travelling time from the district general hospital in Aberdeen, and domiciliary thrombolysis resulted in a time saving of over two hours. This was associated with fewer full thickness $Q$ wave infarctions, better left ventricular function, and a halving of mortality at one year. ${ }^{2}$ Benefits were greatest in patients who received thrombolytic treatment within two hours of the onset of symptoms. An economic analysis showed that the treatment given in GREAT was highly cost-effective. ${ }^{3}$

Recruitment to the trial ran for three years, from 1989 to 1991, and the results were published in 1992. In spite of knowing the results, many of the general practitioners who participated stopped giving thrombolytic treatment when the trial was over. In 1993, only $29 \%$ of respondents to a questionnaire reported giving this treatment in the preceding year, representing $52 \%$ of the practices that had participated in the trial. ${ }^{4}$ The reasons given for not giving this treatment were multiple, but important among them were lack of support from local cardiologists and the need for more training. With the intention of meeting the general practitioners' needs, and with the support of the local cardiologists, an audit of prehospital management of suspected acute myocardial infarction was therefore instigated.

\section{Methods}

In January 1994 a meeting was held for the general practitioners who had participated in GREAT. The latest results from GREAT were presented, together with the guidelines for the early management of patients with myocardial infarction developed by a working group of the British Heart Foundation (BHF). ${ }^{5}$ Those present at the meeting agreed to take part in an audit of their management of patients with suspected acute myocardial infarction, adopting as an audit standard the BHF's call-toneedle time of 90 minutes.

A protocol for the management of patients with suspected acute myocardial infarction was developed, ${ }^{6}$ and a referral letter which prompted the recording of relevant times and included a checklist of the indications and contraindications for thrombolytic treatment was designed and printed. These were distributed during a round of visits in March/April 1994 to all Grampian practices located $\geqslant 30$ minutes' travelling time from Aberdeen and referring their patients to Aberdeen Royal Infirmary. At these visits the importance of early thrombolysis was again stressed, and support of the 
Table 1 Number (\% of available) with various abnormalities of electrocardiograms recorded before admission to hospital in patients given thrombolytic treatment prehospital or in hospital

\begin{tabular}{lcc}
\hline Prehospital ECG & $\begin{array}{l}\text { Thrombolysis } \\
\text { prehospital }\end{array}$ & $\begin{array}{l}\text { Thrombolysis in } \\
\text { hospital }\end{array}$ \\
\hline ST elevation & $108(85)$ & $59(84)$ \\
BBB & $4(3)$ & $3(4)$ \\
Other abnormality & $15(12)$ & $5(7)$ \\
Normal & $0(0)$ & $3(4)$ \\
Available & $127(87)$ & $70(26)$ \\
Not available & $19(13)$ & $198(74)$ \\
Total & $146(100)$ & $268(100)$ \\
\hline
\end{tabular}

$\mathrm{BBB}$, bundle branch block

Table 2 Number (\%) with various discharge diagnoses in patients given thrombolytic treatment prehospital or in hospital

\begin{tabular}{lcc}
\hline Diagnosis & $\begin{array}{l}\text { Thrombolysis } \\
\text { prehospital }\end{array}$ & $\begin{array}{l}\text { Thrombolysis in } \\
\text { hospital }\end{array}$ \\
\hline Definite AMI & $95(65)$ & $226(84)$ \\
Probable AMI & $20(14)$ & $19(7)$ \\
Possible AMI & $11(8)$ & $12(4)$ \\
Angina & $15(10)$ & $8(3)$ \\
Chest pain & $4(3)$ & $3(1)$ \\
Other & $1(1)$ & $0(0)$ \\
Total & $146(100)$ & $268(100)$ \\
\hline
\end{tabular}

AMI, acute myocardial infarction.

cardiologists for the policy of general practitioners giving thrombolytic treatment was emphasised. The protocol for giving thrombolytic treatment was discussed and practical aspects of procuring and using thrombolytic agents were addressed.

The audit began in May 1994 with the appointment of an audit nurse (CS) who identified patients from the participating practices on their admission to hospital, and followed their progress through to discharge; subsequent survival was determined from hospital records. A few patients appeared more than once in the audit. For the purposes of follow up the first recorded episode was taken as the index event, and subsequent episodes were disregarded. Diagnostic criteria were the same as those that had been used in GREAT. ${ }^{1}$

The results of the audit were reported to the practitioners at two practice visits by a consultant (JR), in the winter of $1994 / 5$, and May/June 1996. At these visits practice performance and patients' case records and electrocardiograms were reviewed.

\section{Results}

PREHOSPITAL OR HOSPITAL THROMBOLYTIC TREATMENT

Records were obtained of 806 episodes of suspected acute myocardial infarction; the diagnosis was confirmed on 559 occasions. We identified 414 administrations of thrombolytic treatment in patients initially seen by a general practitioner before being transferred to hospital; these episodes are the basis of this report. In 146 cases $(35 \%)$ thrombolytic treatment was given by the general practitioner (the prehospital group), and in 268 cases (65\%) administration was deferred until after admission to hospital (the hospital group). The proportion of patients receiving thrombolytic treatment from their general practitioners did not vary significantly during the course of the study (1994:
Table 3 Median time intervals for patients given thrombolytic treatment prehospital or in hospital

\begin{tabular}{lcc}
\hline & $\begin{array}{l}\text { Thrombolysis } \\
\text { prehospital }\end{array}$ & $\begin{array}{l}\text { Thrombolysis in } \\
\text { hospital }\end{array}$ \\
\hline Call-to-opiate & 29 & 35 \\
Call-to-thrombolysis & $45(94 \% \leqslant 90)$ & $145(7 \% \leqslant 90)$ \\
Travel & 51 & 45 \\
Door-to-thrombolysis & - & 35 \\
Onset-to-thrombolysis & 150 & 240 \\
\hline
\end{tabular}

Values are times in minutes.

$35 / 92=38 \% ; 1995: 53 / 158=34 \% ; 1996:$ $58 / 164=35 \%)$. The mean ages of prehospital and hospital groups were 63 and 67 years, and men comprised $70 \%$ and $72 \%$. There were 141 and 262 index events in prehospital and hospital groups, respectively.

THE PRESENTING ELECTROCARDIOGRAM

Electrocardiograms recorded prehospital were available for $87 \%$ and $26 \%$ of patients in prehospital and hospital groups (table 1). The proportion of prehospital electrocardiograms showing ST elevation or bundle branch block was identical in the two groups, at $88 \%$. No normal electrocardiograms were identified in the prehospital group, but $4 \%$ of available electrocardiograms in the hospital group were normal when recorded prehospital.

\section{DISCHARGE DIAGNOSES}

Discharge diagnoses for patients given thrombolysis prehospital and in hospital are given in table 2 . The proportion of patients with a final diagnosis of myocardial infarction in any category was $87 \%$ in the prehospital and $95 \%$ in the hospital group, and there were significantly fewer patients with a definite infarction in the former. There was only one patient in the prehospital group with an "other" diagnosis: he had pericarditis, and suffered no adverse events from inappropriate thrombolytic treatment.

\section{DELAY INTERVALS}

Median intervals for call-to-opiate, call-tothrombolysis, travel, and door-to-thrombolysis (hospital group) are given in table 3. Call-toopiate times are similar for prehospital and hospital groups at about half an hour, and travel times are also alike. When thrombolysis was given by general practitioners it was given about a quarter of an hour after the opiate, 45 minutes after the call. The call-to-thrombolysis time in the prehospital group was 100 minutes shorter than in the hospital group. The proportion of patients given thrombolytic treatment within the $\mathrm{BHF}$ target time was $>90 \%$ in the prehospital group and $<10 \%$ in the hospital group. Overall, median onset-tothrombolysis intervals were 2.5 hours and 4.0 hours in prehospital and hospital groups, respectively.

\section{FOLLOW UP}

Audited patients have been followed up for a mean of two years, and Kaplan-Meier survival curves are shown in fig 1 . Estimated survival at one year is better for the prehospital than for the hospital group ( $83 \%$ v $73 \%$; $<0.05$ by log-rank test). 


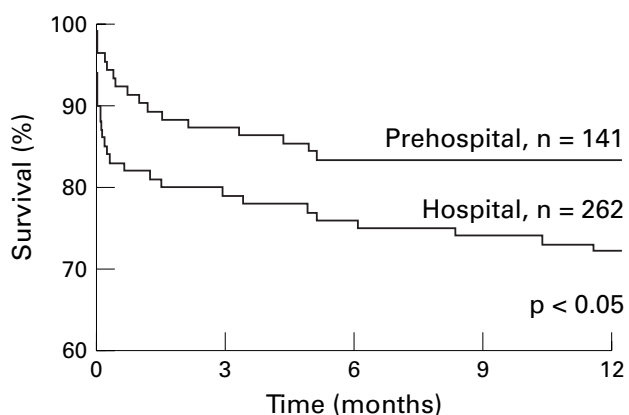

Figure 1 Kaplan-Meier survival curves for patients given thrombolytic treatment prehospital or in hospital.

\section{Discussion}

In GREAT, median call-to-needle times were 55 and 185 minutes for prehospital and hospital groups; $90 \%$ and $1 \%$ of measurements were $\leqslant 90$ minutes, respectively. ${ }^{1}$ In this audit, comprising many of the same practices that took part in the trial, the corresponding times were improved, at 45 minutes for prehospital thrombolysis, and 145 minutes for hospital thrombolysis. The improvement is most marked for the hospital group, where call-toneedle times were 40 minutes shorter in the audit than in the trial. This was largely owing to shortening of door-to-needle times in hospital, from 87 minutes in GREAT (estimated mean), to 35 minutes (median) in this audit.

Call-to-opiate times give an indication of the first opportunity for thrombolysis, which may be initiated about 15 minutes after opiate is given. In both prehospital and hospital groups, median call-to-opiate times were about half an hour.

In some rural areas of Scotland, thrombolytic treatment is given by general practitioners in community hospitals. In a study from the Scottish Association of General Practitioner Community Hospitals, median call-to-needle times of $35(100 \% \leqslant 90), 65(76 \% \leqslant 90)$, and $120(31 \% \leqslant 90)$ minutes were reported for patients given thrombolytic treatment at home, in community hospitals, and in district general hospitals, respectively. ${ }^{7}$ Median call-to-opiate time for all patients, wherever they received thrombolytic treatment, was $25(94 \% \leqslant 90)$ minutes.

These studies show that in communities $\geqslant 30$ minutes' travelling time from a district general hospital, general practitioners-who are most commonly the first medical contact in such areas-have an opportunity in most cases to give thrombolytic treatment within 90 minutes of being called. This audit standard cannot be achieved in the majority of cases unless thrombolytic treatment is given in the community before transfer to hospital. About $10 \%$ of the population of the United Kingdom live in such communities.

During the course of this audit the proportion of patients who received thrombolytic treatment from their general practitioners remained the same, at about $35 \%$. The audit has therefore failed to reach its objective, which was to increase the proportion of eligible patients receiving timely thrombolytic treatment from their general practitioners. Some practices have not adopted a policy of giving thrombolytic treatment, some doctors in practices with such a policy do not adhere to it, and some doctors who give thrombolytic treatment do not give it on all appropriate occasions. General practitioners are diffident over their use of this potent treatment, so it is unlikely that they would ever give thrombolytic treatment to all eligible cases, but would sometimes defer the decision to the hospital coronary care unit. Nevertheless, it was hoped that by the end of the project every general practitioner from the participating practices would have had a policy of giving thrombolytic treatment, and this treatment would have been mostly given by them.

OBSTACLES TO CHANGING CLINICAL PRACTICE

In government departments of health there is a policy vacuum regarding prehospital thrombolysis; early thrombolysis received scant attention in the Scottish Office Department of Health policy review on coronary heart disease, ${ }^{8}$ and the only official public support for general practitioners giving thrombolytic treatment comes from the British Heart Foundation. $^{5}$

In conurbations there are conflicting opinions about the role of general practitioners in the immediate care of patients with suspected acute myocardial infarction, and there is widespread support for a 999 "scoop and run" policy, which is totally inappropriate for peripheral practices. ${ }^{9}$ Some out-of-hours cooperatives have adopted a policy of not attending patients with chest pain, but sending an ambulance instead. ${ }^{10}$ Some cardiologists have expressed reservations about the competence of general practitioners to make the diagnosis of acute myocardial infarction and to interpret an ECG. ${ }^{11}{ }^{12}$ Thus general practitioners in peripheral practices who might wish to use thrombolytic treatment are exposed to these negative attitudes, which have not been countered by any clear directive to the contrary, either nationally or locally.

With an average list size, general practitioners would be expected to use thrombolytic treatment two or three times a year. Injectable thrombolytic agents are expensive (about $£ 500$ per dose), and have a shelf life of two to three years. At a time when general practitioners are being exhorted to contain their prescribing costs, it is unrealistic to expect them to stock an expensive drug that will be used infrequently and might become outdated before use. There are real and perceived financial disincentives to using thrombolytic agents, and these need to be removed.

In Grampian, almost all peripheral practices have a defibrillator and an electrocardiograph. In other regions that may not be so, and lack of this essential equipment constitutes a bar to general practitioners using thrombolytic treatment.

\section{CONCLUSIONS}

About $10 \%$ of people in the United Kingdom live $\geqslant 30$ minutes' travelling time from a district general hospital where thrombolytic 
treatment for acute myocardial infarction is given. Timely thrombolysis can be provided in these areas if it is given in the community before transfer to hospital, but not otherwise. Domiciliary thrombolysis by general practitioners has been shown to be feasible, safe, fast, efficacious, and cost-effective. However, only a minority of eligible patients in peripheral practices receive this life saving treatment from their own doctors. Disincentives to greater use of thrombolytic treatment in these practices relate to the lack of clear policy, negative attitudes of cardiologists, the provision of equipment and drugs, and the need for training. Removal of these disincentives is necessary but may not be sufficient to encourage general practitioners to use this treatment to the full; additional incentives may be required.

This study was funded by Grampian Clinical Audit Committee and Serono Laboratories (UK) Ltd.

1 GREAT Group. Feasibility, safety, and efficacy of domiciliary thrombolysis by general practitioners. $B M \mathcal{F}$ 1992;305:548-53.
2 Rawles J, on behalf of the GREAT Group. Halving of 1-year mortality from acute myocardial infarction by domiciliary thrombolysis in the Grampian region early anistreplase trial (GREAT). $\mathcal{F}$ Am Coll Cardiol 1994;23:1-5.

3 Vale L, Silcock J, Rawles J. An economic evaluation of thrombolysis in a remote rural community. BMF 1997;314: $570-2$.

4 Rawles J. Attitudes of general practitioners to prehospital thrombolysis. BMF 1994;309:379-82.

5 Weston CFM, Penny WJ, Julian DG, on behalf of the British Heart Foundation Working Group. Guidelines for the early management of patients with myocardial infarction. BMF 1994;308:767-71.

6 Rawles J. Guidelines for general practitioners administering thrombolytics. Drugs 1995;50:615-25.

7 Liddell R, Rawles J. Scottish association of general practitioner community hospitals' audit of call-to-needle times in acute myocardial infarction. Occasional paper No 67. Edinburgh: Clinical Resource and Audit Group, The Scottish Office, 1996.

8 Public Health Policy Unit. Coronary heart disease in Scotland. Report of a policy review. Edinburgh: Scottish Office Department of Heath, 1996.

9 Rawles J. Prehospital coronary care. Prehospital Immediate Care 1997; 1:1-8.

10 There's nothing I can do, I'm only a doctor. BMF 1997;314: $759-60$

11 Rule S, Brooksby P, Sanderson J. Use of thrombolysis for acute myocardial infarction by general practitioners. Postgrad Med f 1993;69:190-3.

12 McCrea WA, Saltissi S. Electrocardiogram interpretation in general practice: relevance to prehospital thrombolysis. $\mathrm{Br}$ Heart f 1993;70:219-25. 